\title{
ON BOUNDING THE STÜCKRAD-VOGEL MULTIPLICITY
}

\author{
by L. O'CARROLL
}

(Received 9th June 1989; revised 16th September 1989)

\begin{abstract}
Using a classical result of Nagata, Achilles, Huneke and Vogel gave a criterion for the Stückrad-Vogel multiplicity to take the value one. We use Huneke's extension of Nagata's theorem to give a necessary condition for the Stückrad-Vogel multiplicity to have an arbitrary preassigned bound, under certain conditions. A usable criterion of multiplicity $n$ results (given mild hypotheses). We also revisit some basic results in the Stückrad-Vogel theory in the light of the behaviour of tensor products of affine primary rings, and also revisit some arguments of Achilles, Huneke and Vogel from the point of view of fibre rings.
\end{abstract}

1980 Mathematics subject classification (1985 Revision): Primary 14A05, 14C17. Secondary 13A17, 13 H15.

\section{Introduction}

In [2], Achilles, Huneke and Vogel investigated the situation where the StückradVogel multiplicity takes the value one. The purpose of this note is to extend their main result, to set it in a wider context and to make some additional remarks on some of the arguments in [2].

We first recall the basic context in which the Stückrad-Vogel multiplicity theory operates. To do this, let $K$ be an algebraically closed field and let $X$ and $Y$ be puredimensional subschemes of $n$-dimensional projective space $\mathbb{P}_{K}^{n}$ over $K$. By definition, then, the primary ideals belonging to the homogeneous ideal $I(X)$ of $K\left[x_{0}, \ldots, x_{n}\right]$, which defines $X$, all have the same dimension; a similar statement holds regarding the homogeneous ideal $I(Y)$ of $K\left[x_{0}, \ldots, x_{n}\right]$ which defines $Y$. We consider isolated (or, in other words, irreducible) components $C$ of $X \cap Y$ (i.e. $C$ is defined by an associated prime ideal of the homogeneous ideal $I(X)+I(Y))$ and we focus on the intersection multiplicity of $X$ and $Y$ along $C$.

In the case where $C$ is a proper component (i.e. where $\operatorname{dim} C=\operatorname{dim} X+\operatorname{dim} Y-n$ ) a criterion for this multiplicity to take the value one is given as a statement on transversality (see, e.g. Weil [18, Chapter 6, Theorem 6]). An algebraic version has been given by Nagata [10, (40.6)]; subsequently, the geometrical form was revisited by Fulton [4, Propositions 7.2 and 8.2]. Under the additional hypothesis that the ring concerned should contain a field, Huneke [8] generalized Nagata's algebraic result to give a criterion for bounded Hilbert-Samuel multiplicity (there was an initial important contribution by Ikeda). This additional hypothesis was needed to ensure that the homological conjectures held, and indeed in [11] (where a slightly amended form of Huneke's result is stated) the homological conjectures were seen to enter into Huneke's 
proof in an essential way. For applications of Huneke's theorem, see [8] and [7, pp. 429ff.].

In a parallel development, Achilles, Huneke and Vogel [2] gave a criterion for intersection multiplicity one in the case where the component $C$ is not necessarily proper, using the intersection theory of Stückrad and Vogel. See [2] for further background on such a criterion and for a sketch of this intersection theory; a fuller wellmotivated account is given in [16] (also, cf. [13]). The Stückard-Vogel theory can behave in the "expected" way (see $[15,16]$, for example), but subtleties can arise (see [3, 16], for example). A fascinating discussion of the links between various intersection theories, including those of Stückrad and Vogel and of Fulton and MacPherson, is given in [5].

Note that the dimension of an ideal is, by definition, the Krull dimension of the corresponding factor ring.

This paper, a revised version of the original, has benefitted greatly from helpful and informative discussions with D. Costa, T. Ogoma and N. V. Trung (visiting the U.K.), and (at Halle) R. Achilles, P. Schenzel and W. Vogel-my thanks to them all. Financial support from the Edinburgh and London Mathematical Societies, and from the Royal Society, is gratefully acknowledged.

\section{Stückrad-Vogel multiplicity}

We give a quick précis of some of the main features of Stückrad and Vogel's theory and of those properties of it which are of use here, borrowing heavily from [2] to do so.

Let $X$ and $Y$ be pure-dimensional subschemes of $\mathbb{P}_{K}^{n}$ with defining ideals $I(X)$ and $I(Y)$, respectively, in $K\left[x_{0}, \ldots, x_{n}\right]=: R_{x}$, where $K$ is algebraically closed. Introduce a second copy $K\left[y_{0}, \ldots, y_{n}\right]=: R_{y}$ of $R_{x}$ and denote by $I(Y)^{\prime}$ the ideal in $R_{y}$ corresponding to $I(Y)$. Consider the polynomial ring $R:=K\left[x_{0}, \ldots, x_{n}, y_{0}, \ldots, y_{n}\right]$ and the ideal $c=\left(x_{0}-y_{0}, \ldots, x_{n}-y_{n}\right) R$. Note that $c$ is a prime ideal of height $n+1$ and that it is the kernel of the natural retraction of $R$ onto $R_{x}$ which maps $y_{i}$ to $x_{i}, 0 \leqq i \leqq n$. In fact $c$ is the defining ideal of the diagonal in $A_{K}^{2 n+2}$, which is an irreducible subvariety.

Furthermore, introduce new independent variables $u_{i m}$ over $K, 0 \leqq i, m \leqq n$. Let $\bar{K}$ be the algebraic closure of $K\left(u_{00}, \ldots, u_{n n}\right)$. Put

$$
\bar{R}:=\bar{K}\left[x_{0}, \ldots, x_{n}, y_{0}, \ldots, y_{n}\right] \text { and } l_{i}:=\sum_{m=0}^{n} u_{i m}\left(x_{m}-y_{m}\right)
$$

(in $\bar{R}), 0 \leqq i \leqq n$.

Let $C$ be an isolated component of $X \cap Y$ with defining prime ideal $I(C)$ of dimension $j$. The Stückrad-Vogel theory constructs a well-defined primary ideal belonging to the prime ideal $(I(C)+c) \bar{R}$ such that the intersection multiplicity $j(X, Y ; C)$ of $X$ and $Y$ along $C$ is given by the length of this primary ideal.

Set

$$
\delta:=\text { dimension of } I(X)+I(Y)^{\prime} \text { in } \bar{R}
$$


and $d:=$ dimension of $I(X)+I(Y)$ in $R_{x}$.

Take the linear forms $l_{0}, \ldots, l_{\delta-d-1}$; put

$$
\left(I(X) \bar{R}+I(Y)^{\prime} \bar{R}\right)_{-1}:=I(X) \bar{R}+I(Y)^{\prime} \bar{R}
$$

and

$$
\left(I(X) \bar{R}+I(Y)^{\prime} \bar{R}\right)_{k}:=l_{k} \bar{R}+U\left(\left(I(X) \bar{R}+I(Y)^{\prime} \bar{R}\right)_{k-1}\right),
$$

for $0 \leqq k \leqq \delta-d-1$, where $U(.$.$) is the intersection of all the highest dimensional primary$ components belonging to the ideal (..). Furthermore we put

$$
a_{0}:=U\left(\left(I(X) \bar{R}+I(Y)^{\prime} \bar{R}\right)_{\delta-d-2}\right)
$$

and, if $j<d$,

$a_{s}:=$ intersection of all primary ideals belonging to $U\left(a_{s-1}+l_{\delta-d+s-2} \cdot \bar{R}\right)$ such that $c \bar{R}$ is not contained in their associated primes, $1 \leqq s \leqq d-j$.

Finally, we set

$$
j(X, Y ; C):=\text { length of }\left(a_{d-j}+l_{\delta-j-1} \cdot \bar{R}\right)_{(I(C)+c) \bar{R}}
$$

Then, if $C$ is a proper component, $j(X, Y ; C)$ equals $i(X, Y ; C)$, where the latter is Weil's intersection symbol.

Set

$$
A:=\left(R / I(X)+I(Y)^{\prime}\right)_{I(C)+c}
$$

Then $c A$ is primary to the maximal ideal of the local ring $A$. Most importantly for our purposes, we have the following fact (see [2, p. 53, Remark]):

$$
j(X, Y ; C)=e_{0}(c A, A)
$$

where the latter is the usual Hilbert-Samuel multiplicity symbol.

\section{Remarks}

In this section, we wish to make some remarks about the ring $A$ above and about the corresponding ring

$$
\begin{aligned}
\bar{A}: & =\left(\bar{R} /\left(I(X)+I(Y)^{\prime}\right) \bar{R}\right)_{I(C)+c} \\
& =A \otimes_{K} \bar{K}
\end{aligned}
$$


These rings are the central objects of concern in the basic results [16, (2.3) (and (1.47))] and [2, Lemma 3]. Our aim is to elucidate various aspects of these results and their proofs. We use without mention the basic properties of $\otimes_{K}($ see $[19,20,12]$ for example).

(1). Recall that $I(X)$ is an ideal, all of whose associated primes have the same dimension, and that $I(Y)$ shares analogous properties. Hence $R_{x} / I(X)$ is a subdirect product of affine primary rings $D_{i}$ over $K$, all having the same dimension, and $R_{x} / I(Y)$ is also a subdirect product of affine primary rings $D_{j}$ over $K$, all having the same dimension. Set

$$
A^{\prime}:=\left(R_{x} / I(X)\right) \otimes_{K}\left(R_{x} / I(Y)\right)
$$

it follows that $A^{\prime}$ is a subdirect product of the $D_{i} \otimes_{K} D_{j}$. By Noether's Normalization Lemma, each of these has the same dimension; moreover, each is a primary ring (see [12, Theorem 1] and [19, p. 198, Cor. 1]). Hence $A^{\prime}$ is unmixed in the Stückrad-Vogel (or Zariski-Samuel) sense; i.e. all prime divisors of zero of $A^{\prime}$ have the same dimension. Since

$$
A=A_{\text {I(C)+c }}^{\prime}
$$

the same properties hold for $A$, by the catenary properties of $R$. Thus, using non-reduced structures, $A$ is the local coordinate ring of the join-variety of $X$ and $Y$ in $\mathbb{P}_{K}^{2 n+1}$ at the subvariety defined by $I(C)+c$; the join-variety itself is defined by the homogeneous unmixed ideal $I(X)+I(Y)^{\prime}$. Of course, in the light of $(*)$, this statement is a crucial one. By [20, Chapter VII, $\S 11$ ], similar statements can be made about $\bar{A}$ (cf. [2, pp. 51-53]). Clearly the hypothesis that $K$ be algebraically closed could be weakened to one concerning separability (see [19, p. 195, Theorem 39]).

Geometrically speaking, what we have shown is that the components of the join of $X$ and $Y$ consist of the various joins of the components of $X$ and $Y$ (non-reduced structures again being considered).

It follows from [10, (34.10)] (and the catenary properties of $R$ ) that $A$ and $\bar{A}$ are unmixed in the sense of Nagata (cf. [10, p. 82]); it is unmixedness in the sense of Nagata which enters into his criterion for multiplicity one [loc. cit., (40.6)]. This is a rather unfortunate clash of terminology.

(2). Turning now to the proof of [2, Lemma 3] (see p. 53 there), the authors note that the natural map

$$
\left(R_{x} / I(X)\right)_{I(C)} \rightarrow A
$$

is a local flat homomorphism with fibre $F$ at the maximal ideal, where

$$
F=\left(R /\left(I(C)+I(Y)^{\prime}\right)\right)_{l(C)+c}
$$

This follows quickly on noting that the flat homomorphism $K \rightarrow R_{y} / I(Y)^{\prime}$ yields the flat homomorphism 


$$
R_{x} / I(X) \rightarrow R_{x} / I(X) \otimes_{K} R_{y} / I(Y)^{\prime}=A^{\prime}
$$

by base change.

It will elucidate the proof of [2, Lemma 3], and especially that of the claim on p. 54 there, to remark that, further, for the same reasons as above, there is a local flat homomorphism

$$
\left(R_{y} / I(Y)^{\prime}\right)_{I(C)^{\prime}} \rightarrow F
$$

with fibre $G$ over the maximal ideal, where

$$
G=\left(R /\left(I(C)+I(C)^{\prime}\right)\right)_{I(C)+c}
$$

is a localization of the tensor product of fields

$$
\left(R_{x} / I(C)\right)_{I(C)} \otimes_{K}\left(R_{y} / I(C)^{\prime}\right)_{I(C)^{\prime}}
$$

here, $I(C)^{\prime}$ is (of course) the copy of $I(C)$ in $R_{y}$ and one notes that

$$
I(C)+c=I(C)^{\prime}+c,
$$

since $I(C)^{\prime}$ maps onto $I(C)$ under the natural retraction of $R$ onto $R_{x}$, which has kernel c. Now $G$ is Gorenstein [17; Part II, Proposition 2], and even regular [6; (6.7.4.1)] (again, a hypothesis concerning separability suffices for the latter conclusion); one is now in a position to use standard results on fibres of flat morphisms $[9, \S 23]$. (See [14] for a more general version of the two results quoted above in connection with the structure of $G$. Also, see $[9, \$ 21,23]$ for a brief discussion of related results by Avramov. Related ideas appear in [1].)

\section{Bounding the multiplicity}

We now wish to extend the main theorem of [2]. This we do in the obvious way, by replacing the use of Nagata's criterion $([10,(40.6)])$ in [2] by an appeal to Huneke's theorem [8]. As is clear from [2, pp. 53, 55], the hypothesis that the multiplicity equal one is a very strong one, and in particular implies reducedness of the intersection at the pertinent component. In our more general context, we will have to assume reducedness a priori. The strength of the hypothesis of multiplicity one can be seen as well, in the "classical" context, in the proof of $[10,(40.6)]$.

Before giving the theorem, we introduce the following piece of terminology. A local ring $(B, n)$ is said to be analytically $S_{n}$ if the $n$-adic completion $\hat{B}$ satisfies the Serre $S_{n}$-condition, i.e. if

$$
\text { depth } \hat{B}_{\mathfrak{p}} \geqq \inf (n, \text { height }), \quad \forall p \in \operatorname{Spec} \hat{B} \text {. }
$$


Thus an analytically normal local ring is analytically $S_{2}$. Moreover, as is remarked on [2, p. 55], the ring $A$ above is already analytically $S_{1}$, since this follows from the fact that it is unmixed in the sense of Nagata, which we know is indeed the case (cf. Remark 1 of Section 3 above). Note also, by [9, Theorem 23.9 (iii) and $\S 32]$, that $A$ is analytically $S_{n}$ if and only if it satisfies $S_{n}$.

Theorem. Let $X$ and $Y$ be pure-dimensional subschemes of $\mathbb{P}_{K}^{n}$. Let $C$ be an isolated component of $X \cap Y$ such that $X \cap Y$ is reduced at $C$. Suppose that there exists a positive integer $n$ such that $A$ is $S_{n}$ and $j(X, Y ; C) \leqq n$. Then $\left(R_{x} / I(X)\right)_{I(C)}$ and $\left(R_{x} / I(Y)\right)_{I(C)}$ are Cohen-Macaulay local rings.

Proof. By (*), $j(X, Y ; C)=e_{0}(c A, A)$. Since $X \cap Y$ is reduced at $C$ we have $c A=m$, where $m$ denotes the maximal ideal of $A$. It follows from Huneke's theorem $[8,11]$ (see the above remarks) that $A$ is Cohen-Macaulay, and the result is now immediate from [2, Lemmas 3 and 4$]$.

Remarks. (3) Conversely, if $\left(R_{x} / I(X)\right)_{I(C)}$ and $\left(R_{x} / I(Y)\right)_{I(C)}$ are Cohen-Macaulay, it follows from [2, Lemmas 3 and 4] that $A$ is Cohen-Macaulay and therefore $A$ is $S_{n}$, for all $n$.

(4) Suppose that $\left(R_{x} / I(X)\right)_{I(C)}$ and $\left(R_{x} / I(Y)\right)_{I(C)}$ are normal domains (i.e. suppose $X$ and $Y$ are normal irreducible varieties along $C$ ). As in Remark 1 of Section 3 above, $\left(R_{x} / I(X)\right)_{I(C)} \otimes_{K}\left(R_{x} / I(Y)\right)_{I(C)}$ is a domain (cf. [19, p. 198, Cor. 1]). By [10, (42.10)], it is also a normal domain. Hence its localization $A$ is normal as well, and so is $S_{2}$. In fact, it follows from $[6,(6.7 .3)]$ that if $\left(R_{x} / I(X)\right)_{I(C)}$ and $\left(R_{x} / I(Y)\right)_{I(C)}$ satisfy $S_{n}$ then so does $A$.

\section{REFERENCES}

1. R. ACHILles, On the intersection multiplicity of improper components in algebraic geometry, Beiträge Algebra Geom. 19 (1985), 113-129.

2. R. Achilles, C. Huneke and W. Vogel, A criterion for intersection multiplicity one, Nagoya Math. J. 100 (1985), 49-63.

3. H. FlenNer, On imbedded components of improper intersections in projective space, Arch. Math. 48 (1987), 533-537.

4. W. Fulton, Intersection Theory (Springer-Verlag, Berlin-New York, 1984).

5. L. van Gastel, Excess Intersections (Thesis, Rijksuniversiteit Utrecht, 1989).

6. A. Grothendieck, Éléments de Géometrie Algébrique (Inst. des Hautes Études Sci. (Publ. Math. No. 24), Bures-sur-Yvette, 1965).

7. M. Herrmann, S. Ikeda and U. Orbanz, Equimultiplicity and Blowing-Up (Springer-Verlag, Berlin-New York, 1988).

8. C. Huneke, A remark concerning multiplicities, Proc. Amer. Math. Soc. 85 (1982), 331-332.

9. H. Matsumura, Commutative Ring Theory (Cambridge University Press, Cambridge, 1986).

10. M. Nagata, Local Rings (Interscience Publishers, New York, 1962). 
11. L. O'Carroll, On a theorem of Huneke concerning multiplicities, Proc. Amer. Math. Soc. 99 (1987), 25-28.

12. L. O'Carroll and M. A. Qureshi, Primary rings and tensor products of algebras, Math. Proc. Cambridge Philos. Soc. 92 (1982), 41-48.

13. P. Samuel, Multiplicités des composants excédentaires d'intersection, C.R. Acad. Sci. Paris 228 (1949), 158-159.

14. R. Y. Sharp, Simplifications in the theory of tensor products of field extensions, $J$. London Math. Soc. (2) 15 (1977), 48-50.

15. J. St0ckrad and W. Vogel, An Euler-Poincaré characteristic for improper intersections, Math. Ann. 274 (1986), 257-271.

16. W. Vogel, Lectures on Results on Bézout's Theorem (Lecture Notes, Tata Institute, Springer-Verlag, Berlin-New York, 1984).

17. K. Watanabe, T. Ishikawa, S. Tachibana and $\mathrm{K}$. Otsuka, On tensor products of Gorenstein rings, J. Math. Kyoto Univ. 9 (1969), 413-423.

18. A. Well, Foundations of Algebraic Geometry (Amer. Math. Soc., Providence, R.I., 1946).

19. O. Zariski and P. SAmuel, Commutative Algebra Vol. I (Springer-Verlag, New York, 1958).

20. O. ZARISKı and P. SAmuel, Commutative Algebra Vol. II (Springer-Verlag, New York, 1960).

Department of Mathematics

UNIVERSITY OF EDINBURGH

James Clerk Maxwell Building

Mayfield RoAd

EDINBURGH EH9 3JZ 